\title{
Velocity Field around a Rigid Flapping Wing with a Winglet in Quiescent Water
}

\author{
Srikanth Goli ${ }^{1, *}$, Arnab Roy ${ }^{1}$, Subhransu Roy ${ }^{2}$ \\ ${ }^{1}$ Department of Aerospace Engineering; ${ }^{2}$ Department of Mechanical Engineering, Indian Institute of Technology, \\ Kharagpur, West Bengal, India
}

Received 23 August 2019; received in revised form 07 December 2019; accepted 12 March 2020

DOI: https://doi.org/10.46604/aiti.2020.4674

\begin{abstract}
This study investigated the effect of a winglet on the velocity field around a rigid flapping wing. Two-dimensional particle image velocimetry was used to capture the velocity field of asymmetric one-degree-of-freedom flapping motion. A comparison was conducted between wings with and without a winglet at two flapping frequencies, namely 1.5 and $2.0 \mathrm{~Hz}$. The effect of the winglet on the velocity field was determined by systematically comparing the velocity fields for several wing phase angles during the downstroke and upstroke. The presence of a winglet considerably affected the flow field around the wingtip, residual flow, and added mass interaction. The added mass was lower and residual flow was weaker for the wings with a winglet than for the wings without a winglet. The added mass and velocity magnitudes of the flow field increased proportionally with the flapping frequency.
\end{abstract}

Keywords: square wing, flapping motion, winglet, one-degree-of-freedom flapping, PIV, velocity field

\section{Introduction}

Winglets are employed in modern aircraft for improving aerodynamic performance. Winglets were first proposed by Whitcomb in the 1970s for improving the aerodynamic efficiency by reducing the drag induced [1-2]. Many studies have reported the advantages of different winglet sizes and shapes, especially multiwinglets [3-7]. The winglet concept is based on birds' feathers. Birds such as eagles, hawks, condors, vultures, and ospreys have feathers on their wings [8], which orient them horizontally and vertically in flight to form slotted tips for reducing the induced drag [9]. Winglets attached to wingtips are widely used in commercial and transport aircraft.

In addition to numerous fixed- and rotary-wing aircraft, micro air vehicles (MAVs) have been extensively developed in the last two decades. The flapping-wing MAV (FWMAV) design is gaining popularity due to its many advantages. Natural flyers have various desirable features, such as high maneuverability and perching and hovering capabilities. These features are difficult to achieve in fixed- and rotary-wing aircraft. However, they can be easily achieved in FWMAVs. Thus, FWMAVs are effective for surveillance and reconnaissance missions. FWMAV development has accelerated considerably during the last two decades, and many new technologies have been incorporated into FWMAV design. Similar vehicles used in water-based applications are called micro underwater vehicles. The unsteady flow field around the flapping wings and its effect on the configuration fluid dynamics are not well understood. Detailed understanding of the flow field is essential for improving the efficiency of small-scale flapping-wing vehicles and thus maximizing their endurance.

* Corresponding author. E-mail address: srikanthgoli.ind@ gmail.com 
Qualitative information on the flow field around the flapping wing of insects and mechanical flyers has been reported in [10-13] from studies conducting smoke flow visualization and particle image velocimetry (PIV) studies. Although natural flyers often exhibit flapping motion with multiple degrees of freedom, most studies have focused on one-degree-of-freedom flapping motion. In [14-16], the one-degree-of-freedom flapping motion, force and moments associated with different flapping frequencies, and wing aspect ratios were investigated. Studies should be conducted on simple configurations to obtain a fundamental understanding of complex flow.

To the best of the authors' knowledge, the flow field around a flapping wing with a winglet has not yet been investigated. The present study compared the velocity fields generated by rigid flapping wings with and without a winglet. The motivation of using winglets in a flapping motion was to recreate the motion of natural flyers (with feathers). The advantages associated with winglets have been proven for fixed-wing configurations. The experiments conducted in this study involved one-degree-of-freedom flapping motion that was achieved using a four-bar mechanism. The flapping mechanism was identical to that used in Goli et al. [13]. The velocity field was obtained using two-dimensional PIV measurements. A square planform wing $(\mathrm{AR}=1.0)$ with and without a winglet was studied at two flapping frequencies $(f=1.5$ and $2.0 \mathrm{~Hz})$. PIV measurements were made to capture the velocity field in the mid-chord plane of the flapping wing.

\section{Experimental System}

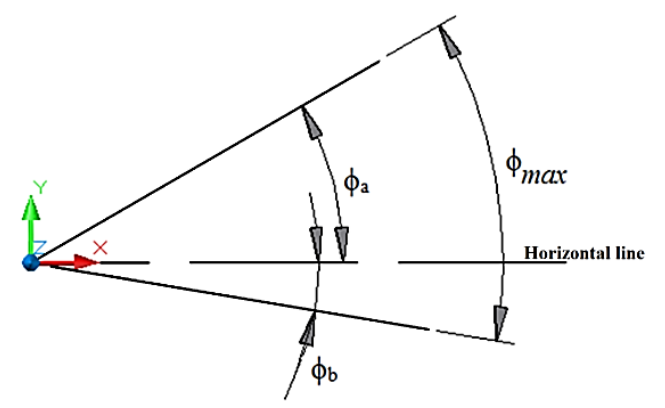

(a) Flapping motion

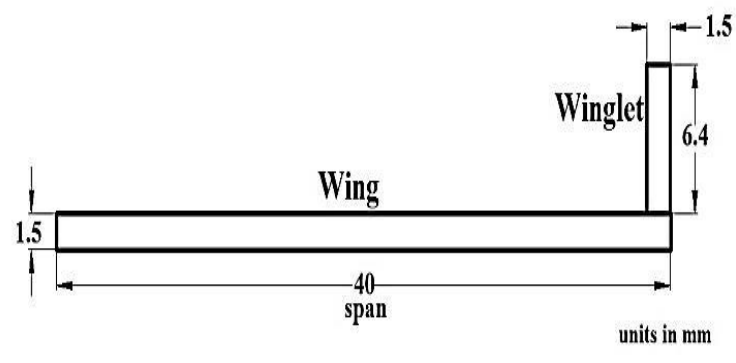

(b) Wing with a winglet (unit: $\mathrm{mm}$ )

Fig. 1 Schematic of the flapping motion of a rigid wing
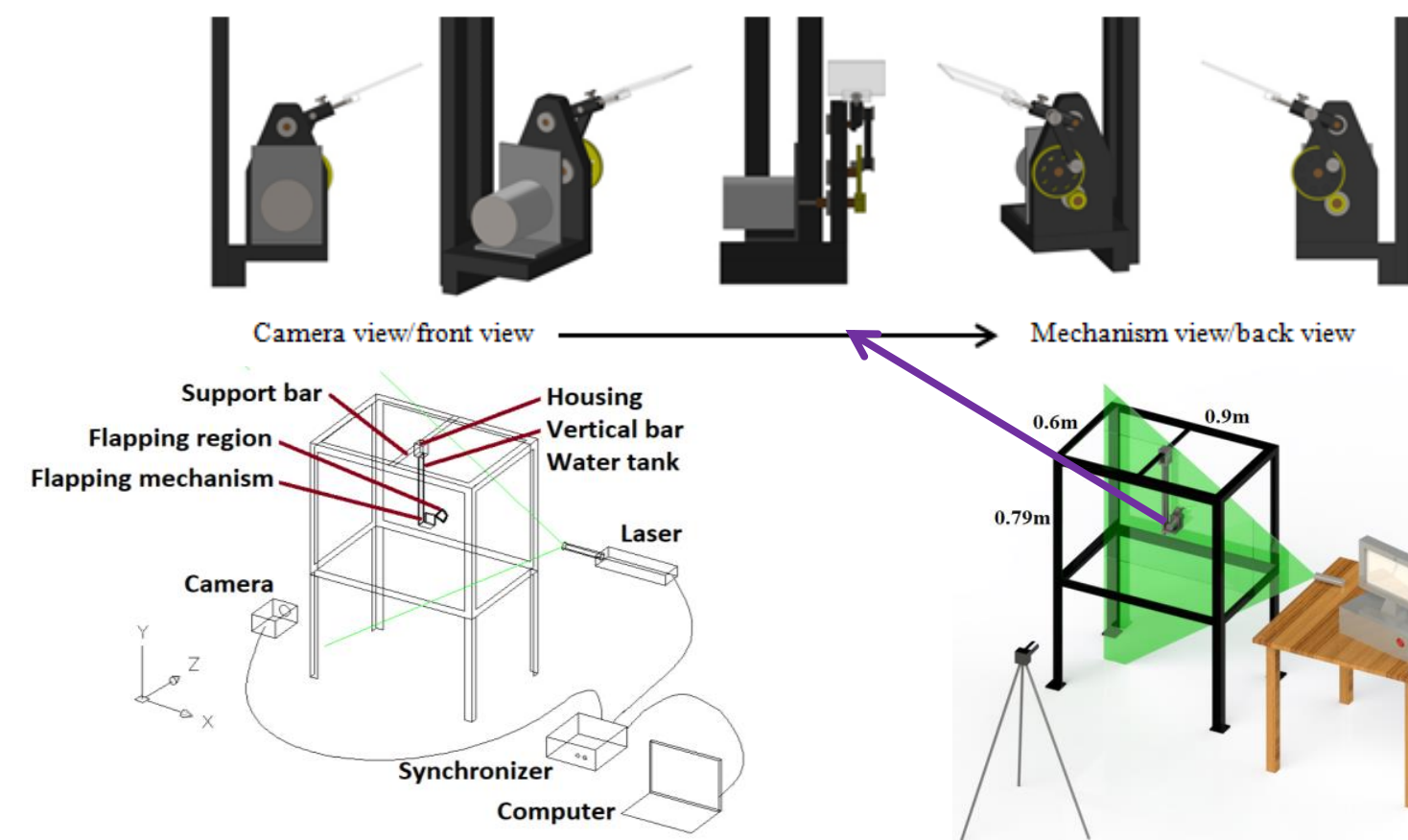

Mechanism view/back view

(a) Parts of the experimental system

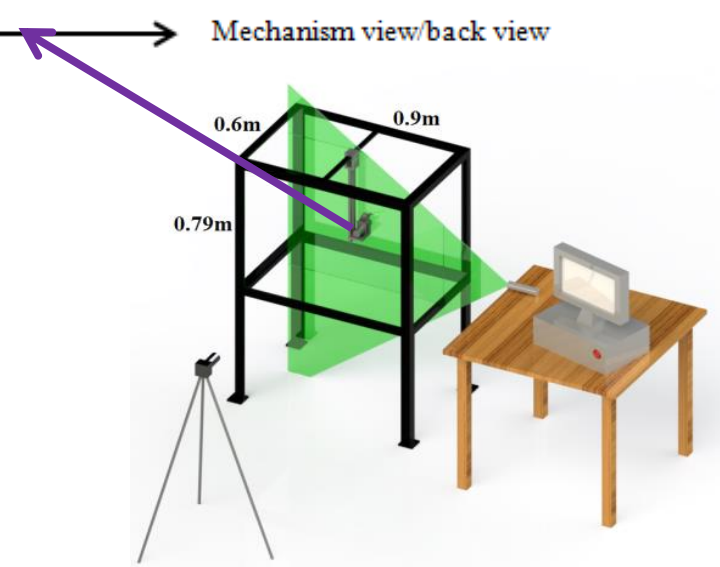

(b) Dimensions of the experimental tank and flapping mechanism

Fig. 2 Schematic of the experimental setup 
Details regarding the flapping mechanism, water tank, fabrication materials, PIV measurements, and experimental procedure are presented in the papers of Goli et al. [17-19]. An overview of the experimental system is presented in the following. In this study, a one-degree-of-freedom flapping motion [Fig. 1(a)] was achieved using a four-bar mechanism (see [13] for dimensions). The wings had a thickness of $1.5 \mathrm{~mm}$ and were made of Perspex acrylic. A schematic of a wing with a winglet is displayed in Fig. 1(b). Experiments were conducted in a water tank in which the flapping mechanism was fully immersed, as displayed in Fig. 2. PIV measurements were performed by illuminating the mid-chord of the wing to capture details of the flow field along the wing span direction. The wing dimensions and operating conditions are presented in Table 1.

Table 1 Wing dimensions and operating conditions

\begin{tabular}{|c|c|c|c|c|c|c|c|c|}
\hline Case & Span $(\mathrm{mm})$ & Chord $(\mathrm{mm})$ & Thickness $(\mathrm{mm})$ & AR & $f(\mathrm{~Hz})$ & Winglet attached & $\phi_{\mathrm{a}}($ degree $)$ & $\phi_{\mathrm{b}}($ degree $)$ \\
\hline I-W & 40 & 40 & 1.5 & 1.0 & 1.5 & yes & 59.5 & -14.5 \\
\hline I & 40 & 40 & 1.5 & 1.0 & 1.5 & no & 59.5 & -14.5 \\
\hline II-W & 40 & 40 & 1.5 & 1.0 & 2.0 & yes & 59.5 & -14.5 \\
\hline II & 40 & 40 & 1.5 & 1.0 & 2.0 & no & 59.5 & -14.5 \\
\hline
\end{tabular}

\section{Results and Discussion}

\subsection{Comparison of the velocity fields for wings with and without a winglet at $f=1.5 \mathrm{~Hz}$ (cases I-W and I)}

Figs. 3 and 4 display the velocity fields during the downstroke and upstroke of the flapping cycle, respectively, for the two cases at $f=1.5 \mathrm{~Hz}$. The parameter $\phi$ represents the flapping angle [Fig. 1(a) and Table 1], whereas $\phi^{*}$ represents the normalized flapping angle. The parameter $\phi^{*}$ varies from 0 to 1 from the beginning to the end of each stroke. The values of the flapping angle $\phi$ and normalized flapping angle $\phi^{*}$ are illustrated in Figs. 3 and 4. The downward and upward arrows in the top right corner of the aforementioned figures indicate downstroke and upstroke, respectively.

In a quiescent fluid, wing flaps do not exhibit cross-flow perpendicular to the PIV image plane. Thus, the vortices and shear produced by the wing are not convected downstream but reside in the region in which the wing flaps. Such reminiscent features form residual flow in the wake of the wing. For example, a wing that executes a downstroke encounters the residual or wake flow formed during the previous upstroke. During an upstroke, the wing encounters the residual or wake flow from the previous downstroke. When a wing flaps through residual or wake flow, the following events occur simultaneously:

(i) The wing recovers energy from energetic structures in the wake flow. This event is termed the wake capture mechanism.

(ii) The wing modifies existing flow structures through strain, rotation, and displacement effects. This process is repeated during wing flapping.

An accelerating or decelerating wing must move some volume of the surrounding fluid with it as it moves. The mass of this dragged fluid is called the added mass, which can be substantial when the wing moves through a heavy fluid. Consequently, a large force must be exerted on the wing to move this additional mass along with the wing mass. The added mass always opposes the accelerating or decelerating motion of the wing.

For both cases illustrated in Fig. 3, a fluid jet was observed at the lower side of the wing that moved in a nearly upward direction at the beginning of the downstroke $\left(\phi=58.50^{\circ}\right)$. When a winglet was used, the residual flow was localized and concentrated below the wing. When no winglet was used, the residual flow was more spread out and angled outward. The residual flow occupied approximately the unit span length for the winglet case and twice the unit span length for the no winglet case. A wingtip vortex formed when no winglet was used but not when a winglet was used.

From $\phi=58.50^{\circ}$ to $\phi=53.50^{\circ}$, the wing traveled through the wake, and the fluid jet continued to move in a nearly vertical direction in both cases. The passage of the wing through the residual flow led to wake capture. No clear wingtip vortex was formed in the winglet case, whereas a clear wingtip vortex was formed in the no winglet case. The wing dragged the fluid on its 
upper surface due to suction. The width of the residual flow was lower in both cases. Specifically, the width of the residual flow was less than the unit span length for the winglet case and less than twice the unit span length for the no winglet case.

When $\phi$ was changed from $53.50^{\circ}$ to $47.50^{\circ}$, the wingtip vortex became visible for the winglet case. In the winglet case, the wingtip vortex emerged from the tip of the winglet. In both cases, the residual flow weakened, moved along the vertical direction, and escaped around the wingtip. Moreover, the strength of the added mass gradually increased around the upper surface of the wing due to the suction induced by the wing movement.

When $\phi$ was $39.50^{\circ}$, the residual flow of the winglet case nearly crossed the lower surface and moved past the wingtip along the vertical direction. In the no winglet case, the residual flow crossed the wingtip, moved vertically with a component directed toward the right, and remained widely spread.

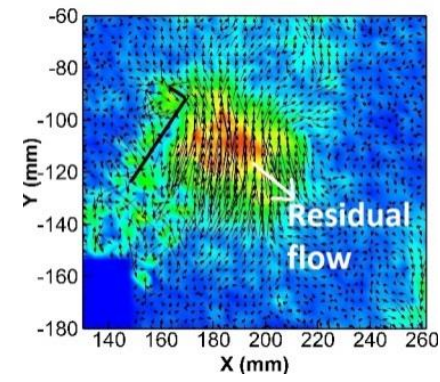

(a) Case I-W when $\phi=58.50^{\circ}$

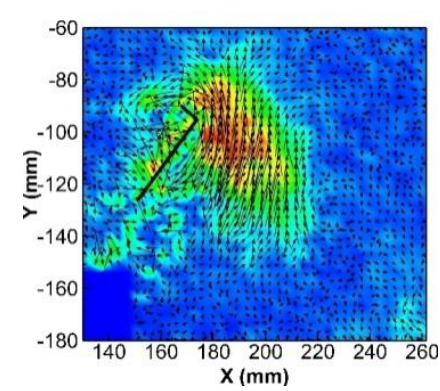

(e) Case I-W when $\phi=53.50^{\circ}$

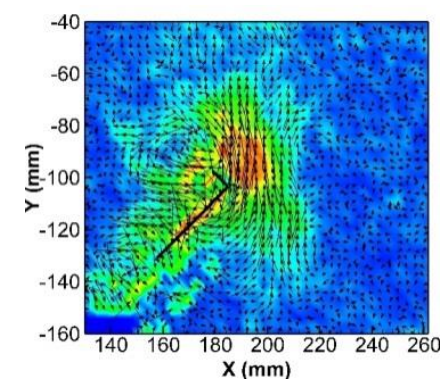

(i) Case I-W when $\phi=44.50^{\circ}$

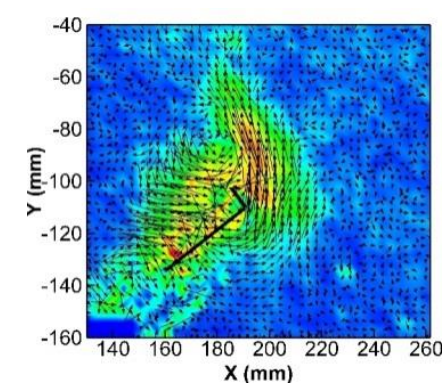

(m) Case I-W when $\phi=39.50^{\circ}$

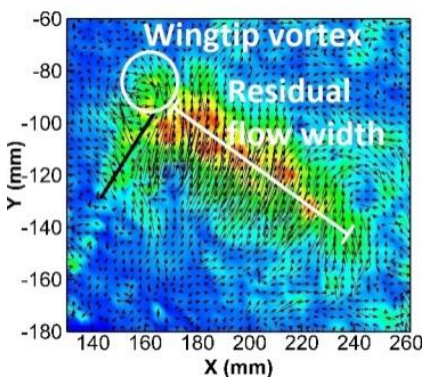

(b) Case I when $\phi=58.50^{\circ}$

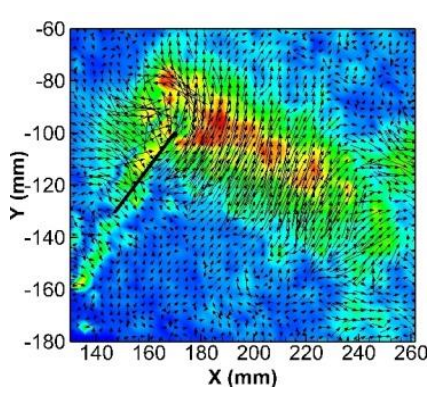

(f) Case I when $\phi=53.50^{\circ}$

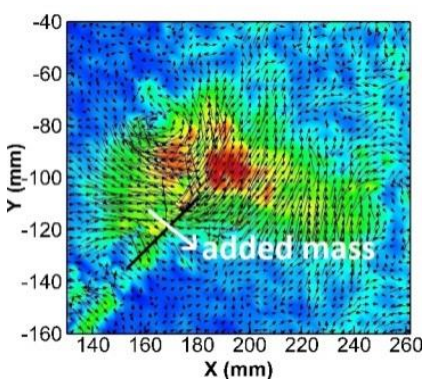

(j) Case I when $\phi=44.50^{\circ}$

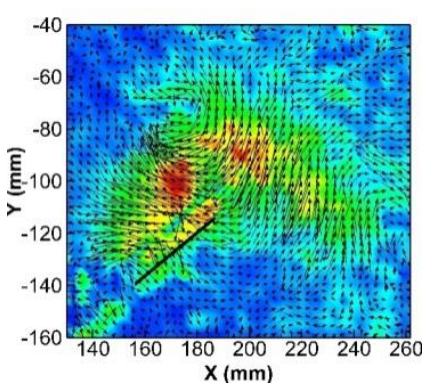

(n) Case I when $\phi=39.50^{\circ}$

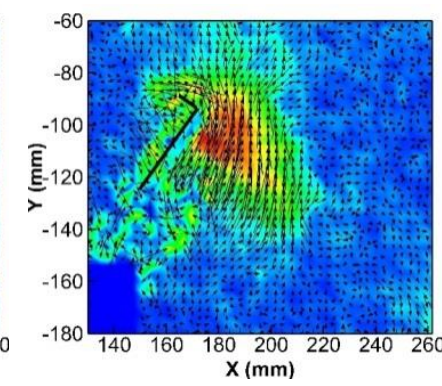

(c) Case I-W when $\phi=55.50^{\circ}$

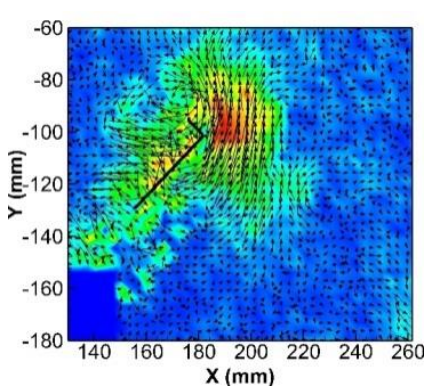

(g) Case I-W when $\phi=47.50^{\circ}$

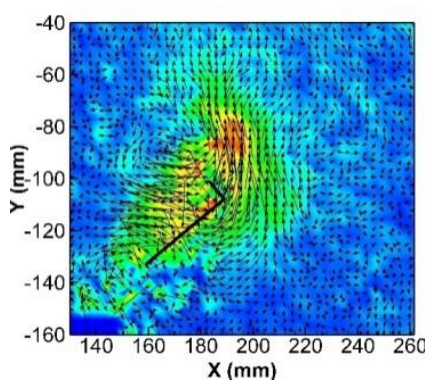

(k) Case I-W when $\phi=41.50^{\circ}$

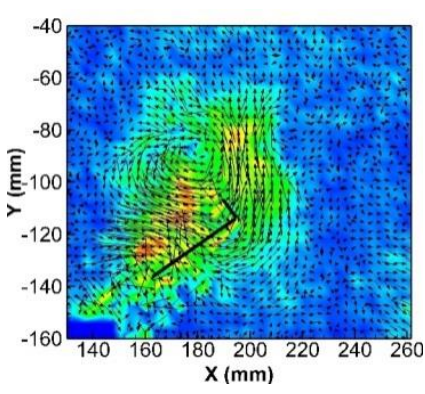

(o) Case I-W when $\phi=36.50^{\circ}$

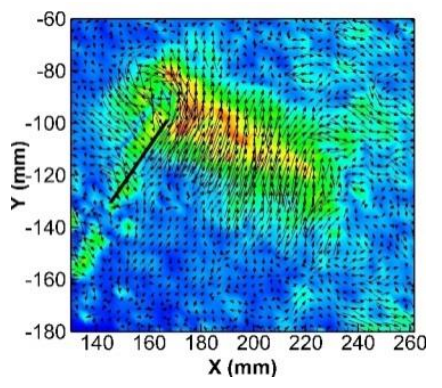

(d) Case I when $\phi=55.50^{\circ}$

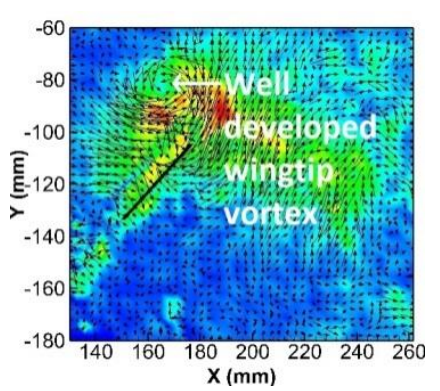

(h) Case I when $\phi=47.50^{\circ}$

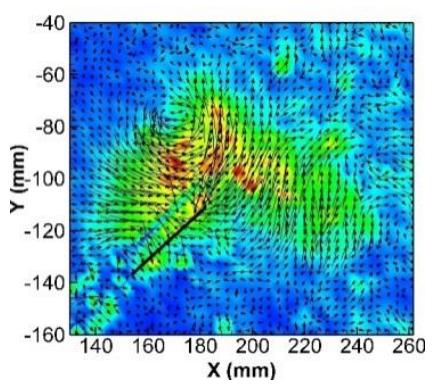

(1) Case I when $\phi=41.50^{\circ}$

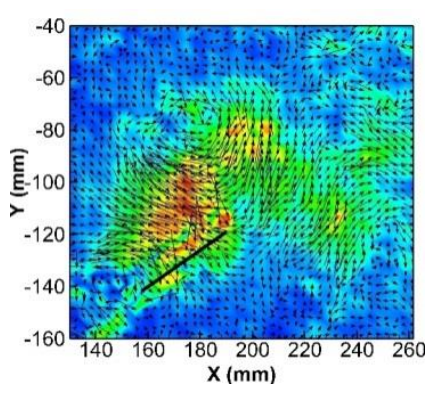

(p) Case I when $\phi=36.50^{\circ}$

$$
\underset{\left(m^{\prime} s\right)}{\mathrm{Vel}} \mathbf{M a g}
$$

0.1

0.3

0.4

0.6

0.7

$\begin{array}{lll}0.8 & 0.9 & 1\end{array}$

Fig. 3 Velocity field for cases I-W and I (with and without a winglet, respectively) when $f=1.5 \mathrm{~Hz}$ during the downstroke 


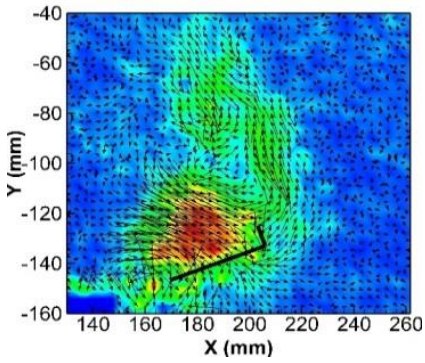

(q) Case I-W when $\phi=22.50^{\circ}$

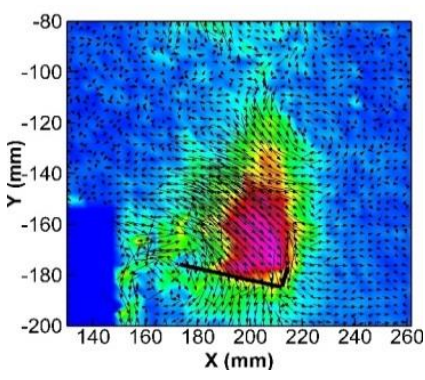

(u) Case I-W when $\phi=-11.50^{\circ}$

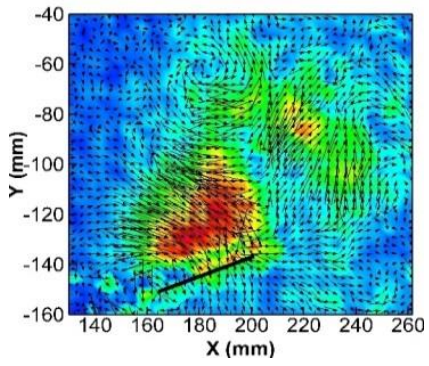

(r) Case I when $\phi=22.50^{\circ}$

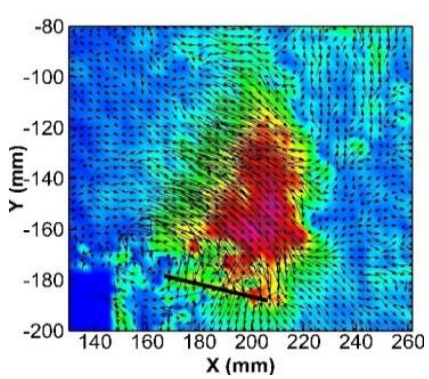

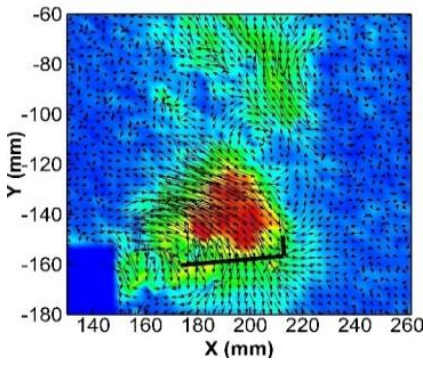

(s) Case I-W when $\phi=07.50^{\circ}$

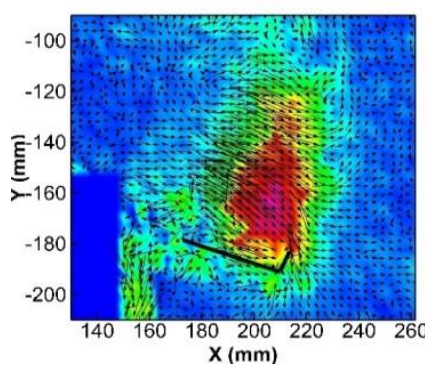

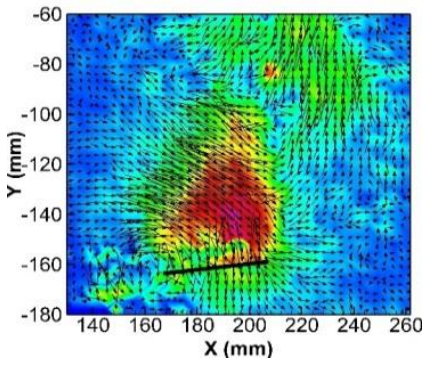

(t) Case I when $\phi=07.50^{\circ}$

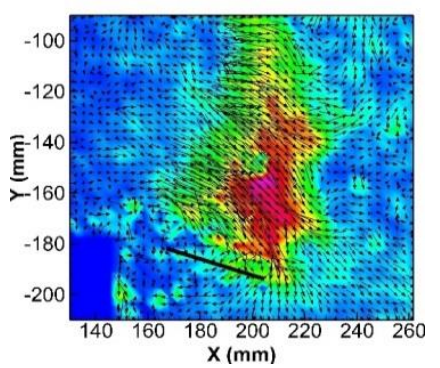

(v) Case I when $\phi=-11.50^{\circ} \quad(\mathrm{w})$ Case I-W when $\phi=-14.50^{\circ}$

$$
\begin{aligned}
& \text { Vel Mag } \\
& \text { (m's) } 0 \\
& 0.1
\end{aligned}
$$

Fig. 3 Velocity field for cases I-W and I (with and without a winglet, respectively) when $f=1.5 \mathrm{~Hz}$ during the downstroke (continued)

The extent of interaction between the residual flow and added mass was influenced strongly by the wingtip geometry. From the beginning of the downstroke, the residual flow was dispersed widely in the no winglet case. The wingtip vortex was detached and located at a distance of approximately one wingspan from the wingtip when $\phi=44.50^{\circ}$. The added mass and residual flow without any hindrance through the gap located between the detached wingtip vortex and the wingtip. The movement of the added mass toward the wingtip diverted the residual flow; thus, the added mass moved vertically with a component directed toward the right. In the winglet case, the added mass stagnated at the inner corner of the winglet. Consequently, a portion of the added mass could not mix with the residual flow.

The added mass above the winglet tip crossed over and joined the residual flow, and the resultant mass moved vertically upward after turning around the outer corner of the wingtip. The impeded interaction between the added mass and residual flow led to a nearly vertical upward-moving residual flow, which was dispersed to a small extent. Thus, in both the cases, the interaction between the added mass formed above the upper surface of the wing and the residual flow below the lower surface of the wing reoriented the wing tip flow. The presence or absence of a winglet considerably affected the flow field around the wingtip, residual flow, and added mass interaction.

In the winglet case, when $\phi$ was $58.50^{\circ}-36.50^{\circ}$, a weak wingtip vortex was formed. This vortex almost maintained its original spatial location with respect to the tip of the winglet. The wingtip flow moved further upward along the outer surface of the winglet. It could not curl around the wingtip because of the presence of the winglet. The wingtip flow energized the shear layer originating from the tip of the winglet and the weak wingtip vortex. These flow features were distinct from those in the no winglet case.

In the winglet case, when $\phi=22.50^{\circ}$, the wingtip vortex was almost completely absent; however, the strength of the shear layer was maintained. The shear layer acted as a barrier between the added mass flow on its inner side above the upper surface of the wing and the wingtip on its outer side. The added mass and wingtip flow moved almost tangentially to the shear layer but had enhanced shear in opposite directions. The presence of the shear layer resulted in less entrainment and mass transfer between the wingtip flow and added mass region. Consequently, the added mass grew to a limited extent in the winglet case. Thus, a strong residual flow led to a strong wingtip flow that considerably influenced the added mass. 
When $\phi$ was $22.50^{\circ}$, the residual flow was weak in the winglet and no winglet cases. Thus, wake capture can be considered to end at $\phi=22.50^{\circ}$. In the winglet case, the interaction between the residual flow and added mass became weaker at angles beyond this angle.

When $\phi$ was $7.50^{\circ}$, no interaction was observed between the residual flow and freshly generated added mass in either case. The added mass in the winglet case was less than that in the no winglet case. From $\phi=7.50^{\circ}$ to the end of the stroke at $\phi=$ $-14.50^{\circ}$, the velocity magnitude in the added mass region increased in both the cases. Pockets were found in the added mass region in which flow curled in the opposite direction due to $\mathrm{KH}$ instability.

A weaker wingtip vortex was formed in the winglet case than in the no winglet case. A strong wingtip vortex consumes considerable energy. If this energy cannot be recovered, the energy lost that must be compensated for through high-energy input from the flapping mechanism. The wingtip vortex is expected to produce a large force on the wing, which improves the wing's lifting capability. However, the aforementioned force can also increase the power consumption. These factors could be the reasons why natural flyers have winglets in the form of feathers in the outboard section of their wings.

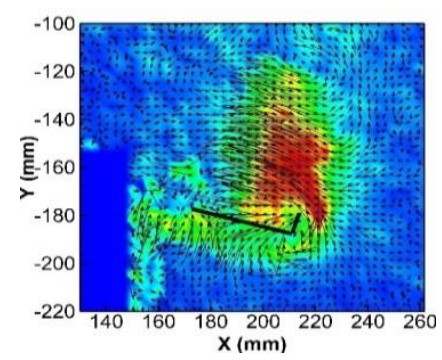

(a) Case I-W when $\phi=-13.50^{\circ}$

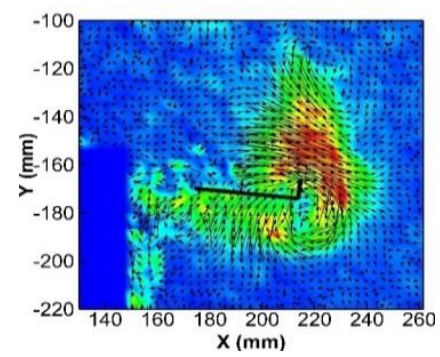

(e) Case I-W when $\phi=-04.50^{\circ}$

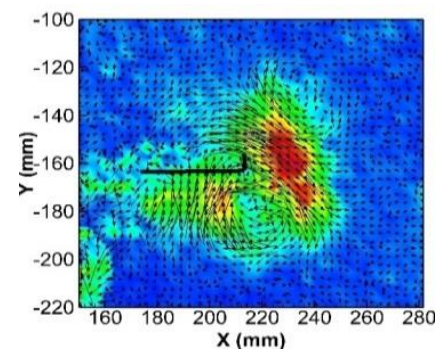

(i) Case I-W when $\phi=03.50^{\circ}$

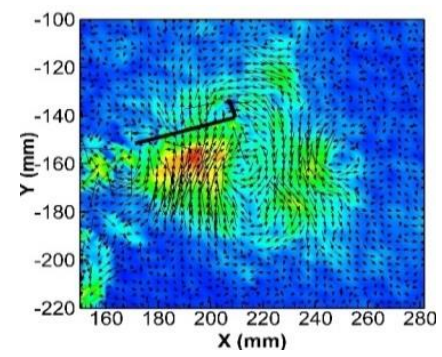

(m) Case I-W when $\phi=17.50^{\circ}$

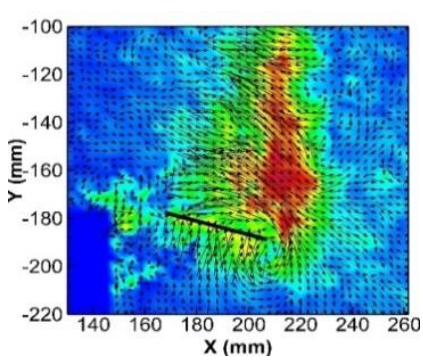

(b) Case I when $\phi=-13.50^{\circ}$

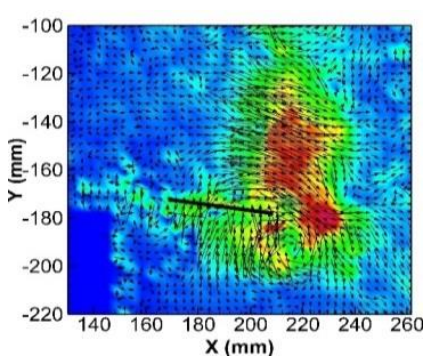

(f) Case I when $\phi=-04.50^{\circ}$

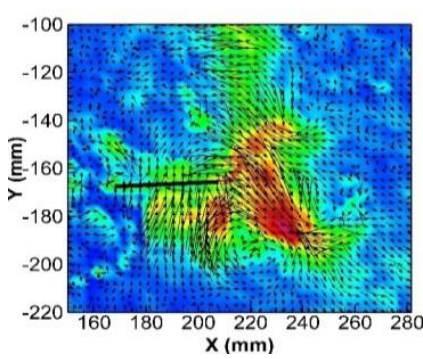

(j) Case I when $\phi=03.50^{\circ}$

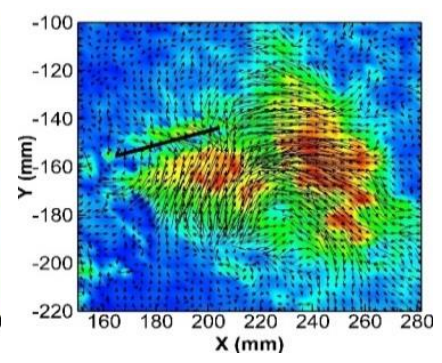

(n) Case I when $\phi=17.50^{\circ}$

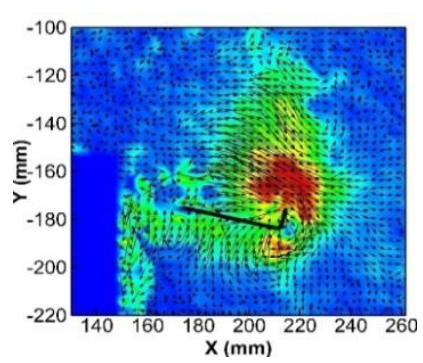

(c) Case I-W when $\phi=-10.50^{\circ}$

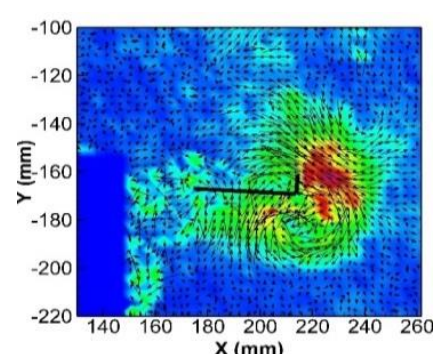

(g) Case I-W when $\phi=-00.50^{\circ}$

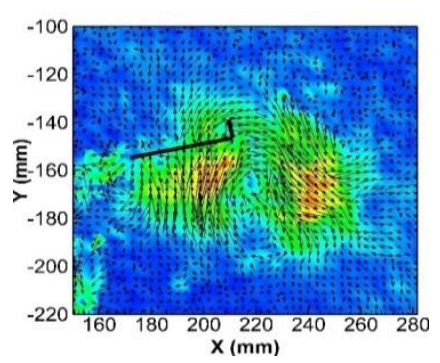

(k) Case I-W when $\phi=13.50^{\circ}$

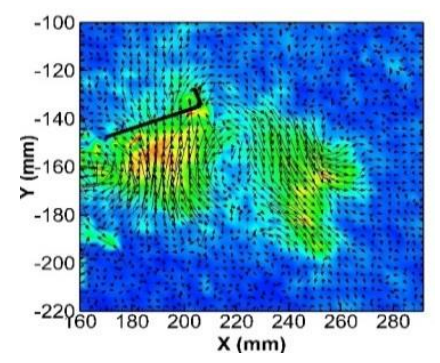

(o) Case I-W when $\phi=21.50^{\circ}$

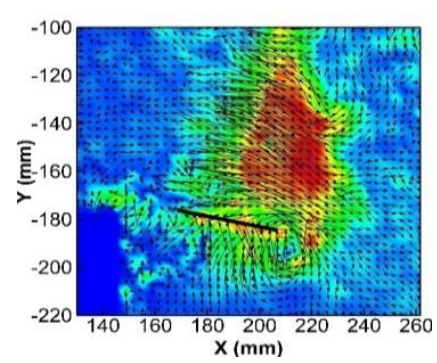

(d) Case I when $\phi=-10.50^{\circ}$

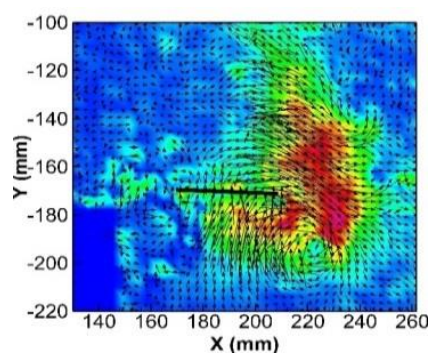

(h) Case I when $\phi=-00.50^{\circ}$

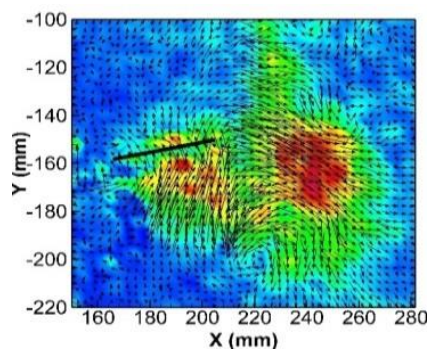

(1) Case I when $\phi=13.50^{\circ}$

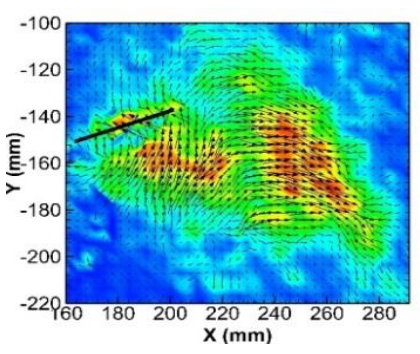

(p) Case I when $\phi=21.50^{\circ}$
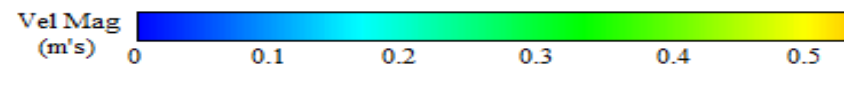

0.6

Fig. 4 Velocity fields for cases I-W and I (with and without a winglet, respectively) when $f=1.5 \mathrm{~Hz}$ during the upstroke 


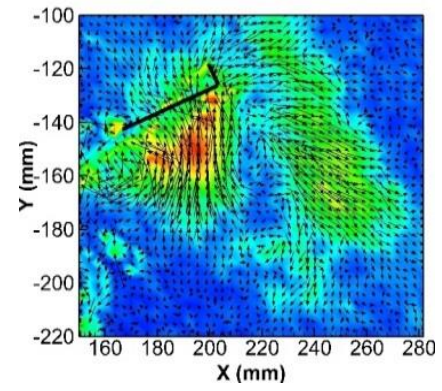

(q) Case I-W when $\phi=27.50^{\circ}$

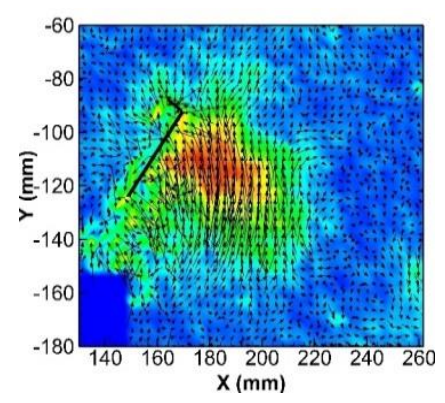

(u) Case I-W when $\phi=57.50^{\circ}$

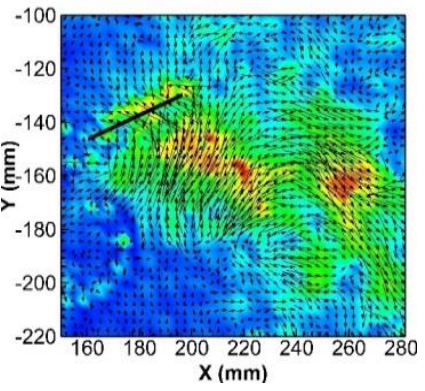

(r) Case I when $\phi=27.50^{\circ}$

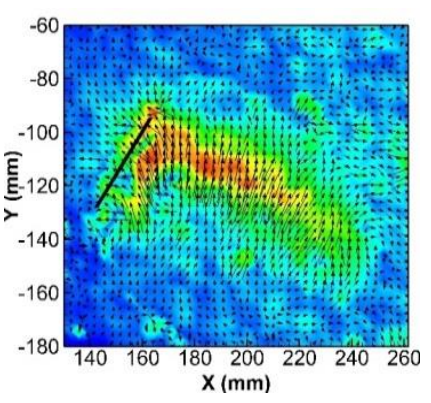

(v) Case I when $\phi=57.50^{\circ}$

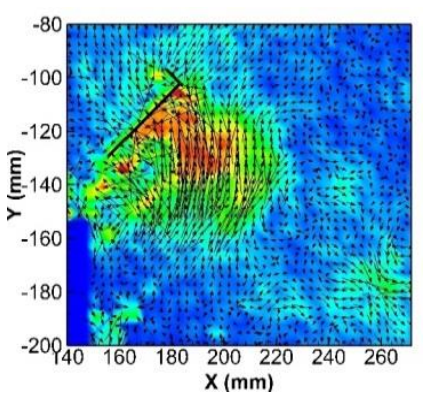

(s) Case I-W when $\phi=46.50^{\circ}$

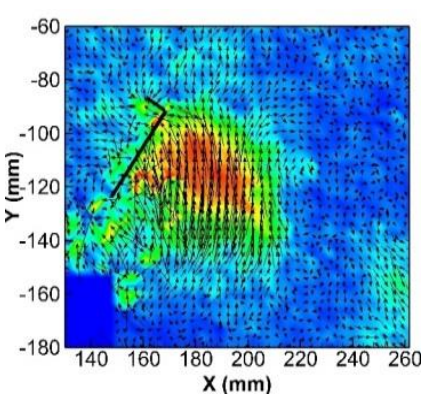

(w) Case I-W when $\phi=59.50^{\circ}$

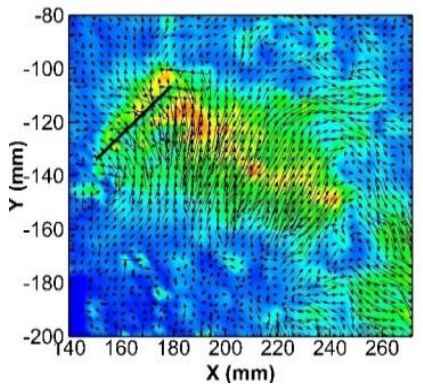

(t) Case I when $\phi=46.50^{\circ}$

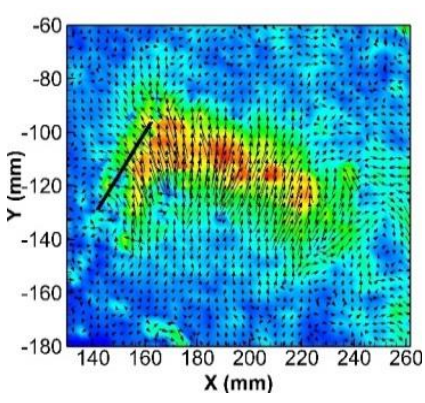

(x) Case I when $\phi=59.50^{\circ}$

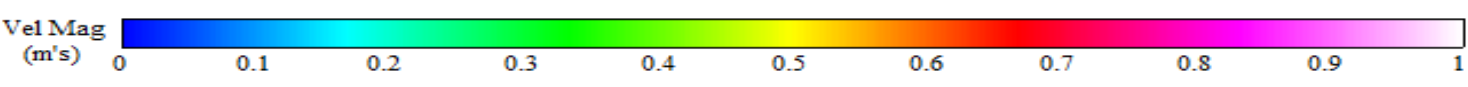

Fig. 4 Velocity fields for cases I-W and I (with and without a winglet, respectively) when $f=1.5 \mathrm{~Hz}$ during the upstroke (continued)

Fig. 4 indicates that at the beginning of the upstroke at $\phi=-13.50^{\circ}$, a wingtip vortex formed in both cases because the residual flow on the upper surface of the wing curled around the wingtip as the wing traveled back (stroke reversal). In the winglet case, the flow crossed over the winglets and formed a wingtip vortex. In the no winglet case, the flow moved smoothly without being impeded. The velocity magnitude in the winglet case was less than that in the no winglet case. The orientation of the residual flow appeared to be nearly horizontal in both cases. The orientation of the residual flow at the beginning of upstroke was different from that of the residual flow at the beginning of the downstroke due to kinematic asymmetry. The residual flow in the winglet case was less than that in the no winglet case, because less added mass was formed in the previous downstroke than in the previous upstroke. The suction effect was initiated on the lower surface of the wing in both cases. This effect resulted in the neighboring fluid being dragged close to the lower surface of the wing. A similar phenomenon occurred during the downstroke.

When $\phi$ was $-10.50^{\circ}$, the wingtip vortex was already enlarged in both cases. Considerably less residual flow occurred in the winglet case than in the no winglet case. In the winglet case, the residual flow on the upper surface of the wing covered approximately $70 \%$ of the wingspan toward the wingtip. In the no winglet case, the coverage of the residual flow was approximately $90 \%$. This result differs from that obtained for the downstroke. In this phase, the wing traveled through its wake and experienced wake capture. The residual flow velocities were low in the winglet case. The added mass increased due to suction on the lower surface of the wing in both cases.

From $\phi=-10.50^{\circ}$ to $\phi=-4.50^{\circ}$, the wingtip vortex continued to grow in both cases. The residual flow decreased in both cases and was located near the wingtip. However, the interaction between the residual flow and wingtip vortex was different in the two cases. In the winglet case, the residual flow was smooth and nearly horizontal because the wingtip vortex was distinct, large, and attached to the wingtip. In the no winglet case, the residual flow curled and acquired a counter-rotating nature because the wingtip vortex was small, was marginally detached, and provided a path for fluid to pass through and interact with the residual flow in the wing region. The residual flow continued to be strong in the no winglet case. In both cases, the wing continued to travel through its wake. 
When $\phi$ was $-0.50^{\circ}$, the wingtip vortex in the winglet case had appearance similar to an inverted six, was large, and was located close to the wingtip. In the no winglet case, the wingtip vortex moved away from the wingtip. The formed wingtip vortex cores were nearly stationary in the winglet case. By contrast, in the no winglet case, the wingtip vortex was shifted along the $x$-axis away from the wingtip. The wing traveled through its wake in both cases. The residual flow in the winglet case was considerably weaker than that in the no winglet case.

The amount of added mass formed due to suction increased in both cases. The added mass in the winglet case was less than that in the no winglet case. During the upstroke, an anchored wingtip vortex played an active role in creating a barrier that restricted the movement of the added mass. A similar phenomenon was observed during the downstroke. The residual flow curled around the wingtip, moved downward along the outer surface of the winglet, and energized the shear layer originating from the wingtip.

When $\phi$ was $03.50^{\circ}$, the wingtip vortex was distorted in both cases due to the shear interaction of the residual flow. The location of the core of the wingtip vortex with respect to the wingtip was almost unchanged in the winglet case, and the wingtip vortex continued to grow close to the wingtip. In the no winglet case, the wingtip vortex moved along the $x$-direction away from the wingtip. The wing continued to travel in its wake. The residual flow in the winglet case was weaker than that in the no winglet case.

When $\phi$ was $13.50^{\circ}$, the wingtip vortex in the winglet case appeared to be stretched similarly to a rubber band and acted as a barrier between the residual flow and added mass, which moved in opposite directions. During the upstroke, the opposite-movement adjacent regions of the flow field energized the shear layer. A similar phenomenon was observed during the downstroke. The barrier effect restricted the movement of the added mass. In the no winglet case, the wingtip vortex was distorted and moved far away from the wingtip. Thus, the added mass in the no winglet case increased, because the suction effect of the moving wing resulted in additional fluid being dragged toward the lower surface of the wing due to entrainment. Residual flow still existed in both cases, and its strength was lower in the winglet case than in the no winglet case. In both cases, the effect of the wake was weakened.

When $\phi$ was $21.50^{\circ}$, the added mass increased further in both cases. The added mass was considerably lower in the winglet case than in the no winglet case due to the barrier effect. Weak residual flow existed far away from the wing. In both cases, the wing did not pass through its wake. This condition was considered the end of wake capture.

When $\phi$ was $27.50^{\circ}$, the added mass in the winglet case was concentrated below the wing due to the barrier effect of the shear layer. In the no winglet case, the added mass bifurcated and moved toward the residual flow located away from the wing surface.

When $\phi$ was $46.50^{\circ}$, the width of the added mass increased in the winglet case. Moreover, the shear layer barrier vanished. Therefore, the added mass had the freedom to move out from the wingtip circumference or wing-sweeping region. In the no winglet case, the width of the added mass increased and it moved out of the wing-sweeping region. No residual flow existed in the winglet case. In the no winglet case, weak residual flow existed far away from the wing and had no influence on the wing and added mass.

From $\phi=46.50^{\circ}$ to the end of the stroke at $\phi=59.50^{\circ}$, the added mass increased and moved vertically in both cases. The added mass was concentrated below the wing in the winglet case but widely spread in the no winglet case.

\subsection{Effect of flapping frequency}

The flow field characteristics for cases I-W and I were compared with those for cases II-W and II to determine the effect of flapping frequency on the flow field characteristics. 
The velocity fields of wings with and without a winglet were compared at two flapping frequencies. The results indicated that the flow features were predominantly similar in the different cases, as discussed in Section 3.1. The added mass and velocity magnitude increased with the flapping frequency. Figs. 5 and 6 depict the velocity fields at the beginning of the downstroke and upstroke, respectively, when $f=1.5$ and $2.0 \mathrm{~Hz}$. The detailed flow field features at discrete flapping angles corresponding to cases II-W and II are not presented here for brevity.

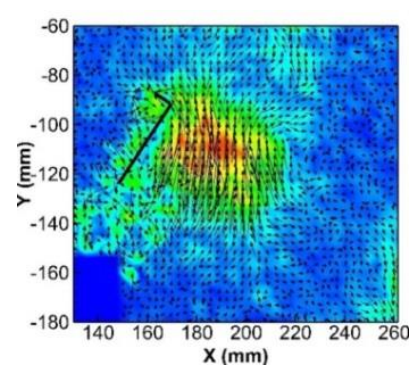

(a) Case I-W

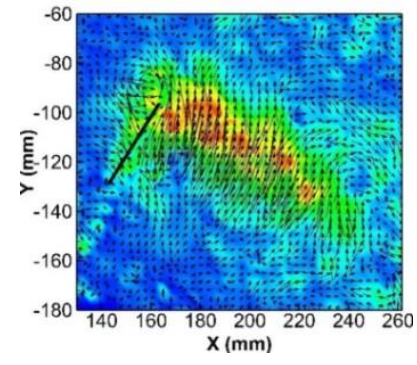

(b) Case I

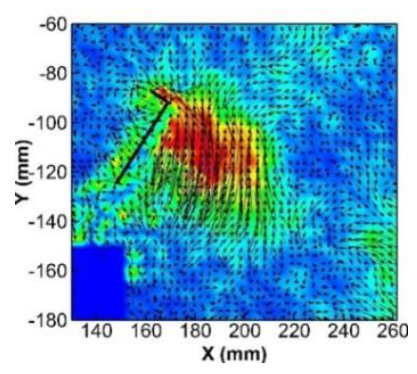

(c) Case II-W

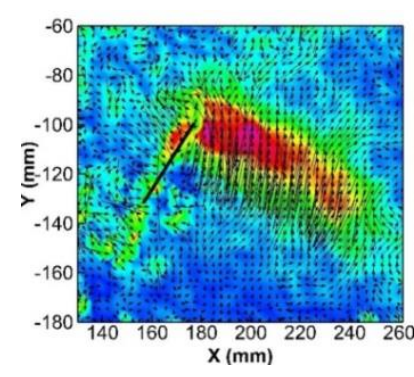

(d) Case II

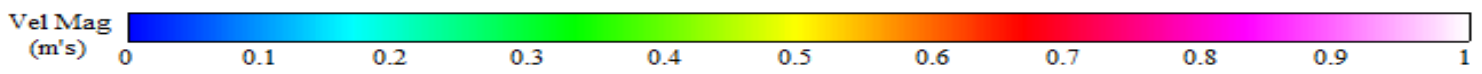

Fig. 5 Comparison of the velocity fields in different cases at the beginning of the downstroke $\left(\phi=58.50^{\circ}\right)$

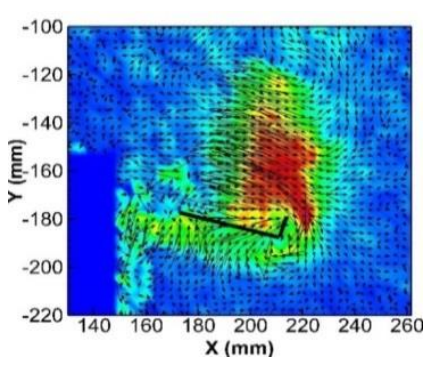

(a) Case I-W

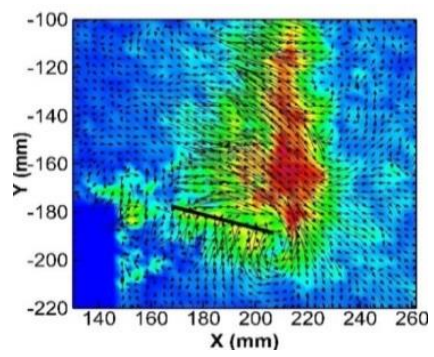

(b) Case I

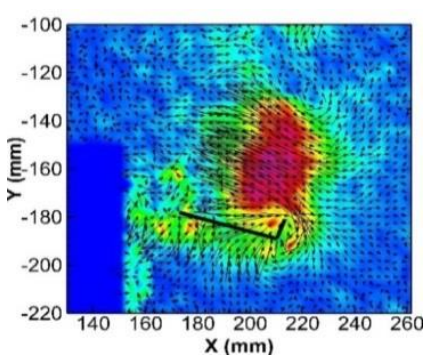

(c) Case II-W

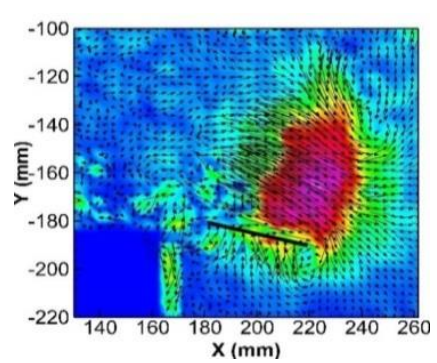

(d) Case II

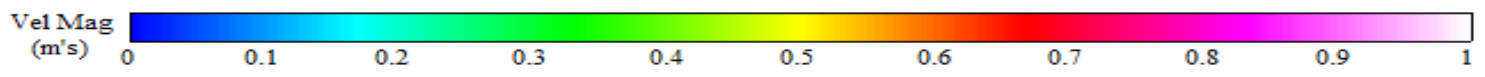

Fig. 6 Comparison of the velocity field in different cases at the beginning of the upstroke $\left(\phi=-13.50^{\circ}\right)$

\section{Conclusions}

Goli et al. described the generic flow field behavior around a rigid flapping wing without a winglet [17-19]. The present study compared the velocity fields of wings with and without a winglet. The effect of the winglet on the velocity field was determined by systematically comparing the velocity fields for several wing phase angles during the downstroke and upstroke.

The effects of the winglet on the velocity field can be summarized as follows:

(1) In the winglet case, the wingtip vortex did not form clearly at the beginning of the downstroke. A weak wingtip vortex formed later in the stroke and appeared to remain anchored to the tip of the winglet. A strong shear layer formed at the tip of the winglet. In the no winglet case, the wingtip vortex formed at the beginning of the downstroke and remained distinct and strong. It detached from the wingtip and created a wide passage for interaction between the added mass and residual flow. During the upstroke, the wingtip vortex was well defined in both cases. In the winglet case, the wingtip vortex remained close to the wing, whereas in the no winglet case, the wingtip vortex detached early and moved far away from the wing.

(2) The added mass was less in the winglet case than in the no winglet case. This result was obtained because the shear layer originating from the tip of the winglet during the downstroke and the wingtip corner during the upstroke reduced entrainment. The reduction in the entrainment impeded the growth of the added mass.

(3) At the beginning of the downstroke, the residual flow in the winglet case was spread close to the wingtip over a distance of approximately one unit span length at the level of the wingtip. In the no winglet case, the residual flow was spread over 
twice the wingspan with an angular orientation of approximately $20^{\circ}$ downward relative to the wingtip level. The residual flow was nearly horizontal at the beginning of the upstroke in both cases. The residual flow in the winglet case was weaker than that in the no winglet case due to the lower added mass formed in the previous downstroke in the winglet case.

(4) The added mass and velocity magnitude of the flow field increased with an increase in the flapping frequency. Overall, the flow features at the two considered frequencies were predominantly similar.

The velocity field has a major effect on wing performance. This behavior must be studied in detail when designing air vehicles. The evolution of vortex structures during wing stroke, kinetic energy variation, added mass, force or torque, strain, rotation and displacement effects, and wing performance will be investigated in a future study to comprehensively determine the effects of a winglet on wing performance.

\section{Acknowledgment}

This research was funded through projects sponsored by the following agencies: (i) the Aeronautics R\&D Board, Government of India (sanction letter no. ARDB/01/1031864/M/I), and (ii) the Department of Science and Technology, Government of India [sanction letter no. SR/FST/ETI-386/2014 (G)].

\section{Conflicts of Interest}

The authors declare no conflicts of interest.

\section{References}

[1] R. T. Whitcomb, “A design approach and selected wing-tunnel results at high subsonic speeds for wing-tip mounted winglets," Washington, United States, Technical Report NASA TN D-8260, July 01, 1976.

[2] S. G. Fletcher and P. F. Jacobs, R. T. Whitcomb, "A high subsonic speed wind-tunnel investigation on a representative second-generation jet transport wing," Washington, United States, Technical Report NASA TN D-8264. July 01, 1976.

[3] I. Kroo, Drag due to lift: Concepts for prediction and reduction, Annual Review of Fluid Mechanics, 2001.

[4] H. D.Cerón-Muñoz and F. M. Catalano, "Experimental analysis of the aerodynamic characteristics adaptive of multi-winglets," The Proceedings of the Institution of Mechanical Engineers, Part G: Journal of Aerospace Engineering, vol. 220, no. 3, pp. 209-215, March 2006.

[5] D. P. Coiro, F. Nicolosi, F. Scherillo, and U. Maisto, "Design of multiple winglets to improve turning and soaring characteristics of angelo d' arrigo's hang-glider: Numerical and experimental investigation” In: XIX Conggresso Nazionale AIDAA 17-21 September 2007 Aerotecnica Missili e Spazio., vol. 87, no. 2, pp. 74-85, May 2008.

[6] M. Berens, "Potential of multi-winglet systems to improve aircraft performance," Doctoral Thesis, Technical Univ of Berlin, Germany, 2008.

[7] G. Srikanth and B. Surendra, "Experimental investigation on the effect of multi-winglets," International Journal of Mechanical and Industrial Engineering, vol. 1, no. 1 pp. 43-46, January 2011.

[8] M. J. Smith, N. Komerath, R. Ames, O. Wong, and J. Pearson, "Performance analysis of a wing with multiple winglets," In: 19th AIAA Applied Aerodynamics Conference, Anaheim, CA, USA, June 2001, pp. 1-10.

[9] V. A. Tucker, "Gliding birds: Reduction on induced drag by wing tip slots between the primary feathers," The Journal of Experimental Biology, vol. 180, no. 1, pp. 285-310, July 1993.

[10] K . Kitagawa, M. Sakakinara, and M. Yasuhara. "Visualization of flapping wing of the drone beetle," Journal of Visualization, vol. 12, no. 4, pp. 393-400, December 2009.

[11] Y. Liu, B. Cheng, and X. Deng. "An application of smoke-wire visualization on a hovering insect wing," Journal of Visualization, vol. 16, no. 3, pp. 185-187, Auguest 2013.

[12] H. Ren, Y. Wu, and P.G. Huang. "Visualization and characterization of near-wake flow fields of a flapping-wing micro air vehicle using PIV," Journal of Visualization, vol. 16, no. 1, pp. 75-83, February 2013.

[13] S. Goli, A. Roy, D. K. Patel, and S. Roy, Particle image velocimetry measurements of rigid and flexible rectangular wings undergoing main flapping motion in hovering flight, Fluid Mechanics and Fluid Power-Contemporary Research, 2017.

[14] Y. S. Hong and A. Altman, "Lift from spanwise flow in simple flapping wings," Journal of Aircraft, vol. 45, no. 4, pp. 1206-1216, July 2008. 
[15] H. Hu, A. G. Kumar, G. Abate, and R. Albertani, “An experimental investigation on the aerodynamic performances of flexible membrane wings in flapping flight," Aerospace Science and Technology, vol. 14, no. 8, pp. 575-586, December 2010.

[16] K. Mazaheri and A. Ebrahimi, "Experimental study on interaction of aerodynamics with flexible wings of flapping vehicles in hovering and cruise flight," Archieve of Applied Mechanics, vol. 80, no. 11, pp. 1255-1269, November 2010.

[17] S. Goli, A. Roy, and S. Roy, "Vortex filamentation and fragmentation phenomena in flapping motion and effect of aspect ratio and frequency on global strain, rotation and enstrophy," International Journal of Micro Air Vehicles, vol. 11, pp. 1-30, January 2019.

[18] S. Goli, S. S. Dammati, A. Roy, and S. Roy, "Coherent structures in the flow generated by rigid flapping wing in hovering flight mode," IOP Conference Series: Materials Science and Engineering, vol. 402, no. 1, pp. 1-18, 2018.

[19] S. Goli, S. S. Dammati, A. Roy, and S. Roy, "Vortex identification and proper orthogonal decomposition of rigid flapping wing,” International Journal of Fluid Mechanics Research (accepted). 2019.

Copyright $\odot$ by the authors. Licensee TAETI, Taiwan. This article is an open access article distributed under the terms and conditions of the Creative Commons Attribution (CC BY-NC) license (https://creativecommons.org/licenses/by-nc/4.0/). 